\title{
PREFACE: INTERNATIONAL WORKSHOP ON HEAT TRANSFER ADVANCES FOR ENERGY CONSERVATION AND POLLUTION CONTROL (IWHT-2013) PART 2
}

Over the last few decades, there has been an increasing worldwide effort to extensively embrace energy-efficient, cost-effective, renewable, and relatively cleaner technologies in a variety of energy conversion and pollution control devices and processes. In all energy-related systems, heat transfer plays a key role and typical applications include heat exchangers, gas turbines, electrical machines, computers and electronic devices, fuel cells, solar energy harvesting and conversion systems, biofuel-producing reactors and combustors, etc.

To provide an international platform for exchange of related scientific information and the latest technological development, with focus on recent advances on heat and/or mass transfer problems, the Second International Workshop on Heat Transfer Advances for Energy Conservation and Pollution Control (IWHT-2013) was held October 18-21, 2013 in Xi'an, China. This was a continuation from the first IWHT held in 2011 also in Xi'an. The hosts were the Key Laboratory of Thermo-Fluid Science and Engineering, Ministry of Education, Xi'an Jiaotong University, China, and its sponsors were the National Natural Science Foundation of China, Chinese Society of Engineering Thermophysics, International Center for Heat and Mass Transfer, and Xi'an Jiaotong University. The International Scientific and Advisory Committee was chaired by Professors Wen-quan Tao (China), Bengt Sundén (Sweden), Afshin Ghajar (USA), and Petr Stehlik (Czech Republic). Over 180 researchers from 15 countries gave a large set of technical presentations, supplemented by several plenary and keynote lectures, covering broad issues relating to the oil and chemical engineering industry, engines and gas turbines, fuel cells, solar energy, aerospace, etc., all of which can be found in the Proceedings of IWHT-2013.

From the workshop presentations, based on their relevance and significance, several authors were invited to submit updated and extended manuscripts, the final selected papers cover interesting topics presented at IWHT-2013. The papers concern numerical investigation of inverse problems, impinging jets of nanofluids, Joule-Thomson effect for $\mathrm{CO}_{2}$ leakage, second law analysis of loss coefficient in conduit bends, conjugated heat transfer analysis of film cooling with relevance to gas turbines, interface effects in an enclosure partially filled with a porous media, stability of flows affected by a magnetic field, and finally temperature fluctuation characteristics for pool boiling issues.

We thank the authors for their contributions and efforts and we hope the readers will find the papers of interest.

Also, the guest editors would like to acknowledge and thank the invaluable assistance provided by Drs. Jian Yang and Ting Ma (IWHT-2013 Secretariat) and several students at Xi'an Jiaotong University.

Guest Editors:

Bengt Sunden, Lund University,

Lund, Sweden

Yitung Chen, University of Nevada, Las Vegas, NV, USA

\author{
Qiuwang Wang, \\ Xi'an Jiaotong University, \\ Xi'an, China \\ Zhixiong Guo, \\ Rutgers, the State University of New Jersey, \\ New Brunswick, NJ, USA
}

